\title{
Induction of Pluripotency in Adult Equine Fibroblasts without c-MYC
}

\author{
Khodadad Khodadadi, ${ }^{1}$ Huseyin Sumer, ${ }^{1}$ Maryam Pashaiasl, ${ }^{1,2}$ Susan Lim, ${ }^{3}$ \\ Mark Williamson, ${ }^{4}$ and Paul J. Verma ${ }^{1,5}$ \\ ${ }^{1}$ Centre for Reproduction and Development, Monash Institute of Medical Research, Monash University, Clayton, VIC 3800, Australia \\ ${ }^{2}$ Faculty of Medicine, Tabriz University of Medical Sciences, Tabriz 51666-14766, Iran \\ ${ }^{3}$ Stem Cell Technologies i (SCTi), Gleneagles Medical Centre, Singapore 258499 \\ ${ }^{4}$ Gribbles Veterinary, Clayton, VIC 3168, Australia \\ ${ }^{5}$ South Australian Research Institute (SARDI), Turretfield Research Centre, Rosedale, SA 5350, Australia
}

Correspondence should be addressed to Huseyin Sumer, huseyin.sumer@monash.edu

Received 9 November 2011; Revised 28 December 2011; Accepted 3 January 2012

Academic Editor: Rajarshi Pal

Copyright ( $) 2012$ Khodadad Khodadadi et al. This is an open access article distributed under the Creative Commons Attribution License, which permits unrestricted use, distribution, and reproduction in any medium, provided the original work is properly cited.

\begin{abstract}
Despite tremendous efforts on isolation of pluripotent equine embryonic stem (ES) cells, to date there are few reports about successful isolation of ESCs and no report of in vivo differentiation of this important companion species. We report the induction of pluripotency in adult equine fibroblasts via retroviral transduction with three transcription factors using OCT4, SOX2, and KLF4 in the absence of c-MYC. The cell lines were maintained beyond 27 passages (more than 11 months) and characterized. The equine iPS (EiPS) cells stained positive for alkaline phosphatase by histochemical staining and expressed OCT4, NANOG, SSEA1, and SSEA4. Gene expression analysis of the cells showed the expression of OCT4, SOX2 NANOG, and STAT3. The cell lines retained a euploid chromosome count of 64 after long-term culture cryopreservation. The EiPS demonstrated differentiation capacity for the three embryonic germ layers both in vitro by embryoid bodies (EBs) formation and in vivo by teratoma formation. In conclusion, we report the derivation of iPS cells from equine adult fibroblasts and long-term maintenance using either of the three reprogramming factors.
\end{abstract}

\section{Introduction}

Cartilage and tendon injuries are common features of tissue damage in both humans and horses. These two tissues have a poor vascular system with low mitotic ability and therefore a limited ability for self-repair. The reduced performance and reinjury create considerable attention for treatments [1].

Adult mesenchymal stem cells (MSCs), embryonic stem cells (ESCs), and reprogrammed somatic cells such as induced pluripotent stem (iPS) cells can provide potential sources of cells for treatment of cartilage and tendon injuries.

MSCs can be isolated from different sources such as bone marrow aspirates [2], umbilical cord [3], and adipose tissue [4] and have the ability to differentiate into different cell types such as muscle, cartilage, and bone [5-7]. MSCs have been used for treatment of cartilage injuries in equines and humans. Although there were the early improvements in cartilage injuries, no significant or long-term recovery could be observed $[8,9]$. In addition, MSCs are limited in bone marrow aspirates and need to be cultured after isolation for at least 4 weeks and have limited in vitro differentiation potential compared with ESCs $[1,10]$.

ES cells can overcome this limitation, as they can provide an inexhaustible supply of cell derivatives of all three germ layers. Despite tremendous efforts on isolation of ESCs, to date there are a few reports on isolation of equine ESCs, which had limited success and no investigation of in vivo differentiation of the isolated cells $[11,12]$. Isolation of equine ESCs is difficult due to the shortage of oocytes and embryos, as well as complexity associated with oocyte collection, maturation, IVF, and in vitro culture in this species [13]. Even if ES cells can be successfully derived, a subsequent problem is the anticipated immune rejection of the derivatives of 
ES cells by the recipient due to incompatibility of the major histocompatibility complex (MHC) antigens because of differences in genomic DNA compared with that of the recipient [14].

There are alternative methods to produce autologous cells lines via reprogramming of adult somatic cells to the pluripotent states such as somatic cell nuclear transfer (SCNT) $[15,16]$ and induced pluripotent stem (iPS) cells [17]; however, limitation with derivation of equine ESCs extend to SCNET-ESC isolation as well.

Takahashi and Yamanaka [17] reported the generation of pluripotent cells from adult mouse fibroblast following retroviral-mediated transduction of four transcription factors, OCT4, SOX2, $c-M Y C$, and KLF4. A number of studies have shown that iPS cells are similar to ESCs in morphology and epigenetic status, expression of pluripotent markers, and ability to differentiate into derivatives of all three embryonic germ layers both in vivo and in vitro and contribute to the germ-line in chimeric mice confirming their true pluripotency [17-19]. Therefore, these cells could have therapeutic application in both human and animals.

Pluripotency has been induced in somatic cells from human [20], primate [21], rat [22, 23] pigs [24-27], sheep [21], and cattle [28].

More recently, the generation of equine iPS cell lines from fetal fibroblasts using transposon-based delivery of four factors has been reported [29]. In this study, we report the generations of equine-induced pluripotent stem (EiPS) cells by retroviral-mediated transduction of adult equine fibroblasts using three transcription factors: OCT4, SOX2, and KLF4, (OSK) without the protooncogene $c-M Y C$, and the pluripotent characteristics of the resulting EiPS cells have been demonstrated both in vitro and in vivo.

\section{Materials and Methods}

Experimental procedures were carried out under the guidelines of the Monash University, Animal Ethics Committee, and conducted according to the International guidelines for Biomedical Research Involving Animals. All chemicals were sourced from Sigma (Castle Hill, Australia) unless otherwise stated.

\subsection{Generation of Induced Pluripotent Stem (iPS) Cells from Adult Equine Fibroblasts}

2.1.1. Transfection, Isolation, and Culture of iPS Cells. Equine iPS cells were generated as previously reported [28]. Briefly, for VSVG pseudotyped retroviral production $3 \times 10^{6}$ GP2 293 cells (Clontech; Scientifix, Cheltenham, Australia) were seeded in a $100 \mathrm{~mm}$ culture dish one day before transfection and incubated overnight at $37^{\circ} \mathrm{C}, 5 \% \mathrm{CO}_{2}$. pMX-based retrovirus vectors encoding human DNA sequence of OCT4, $S O X 2$, and KLF4 were transfected into packaging cells (GP2 293) by FuGENE 6 transfection reagent (Roche, Castel Hill, Australia), and the media were replaced by fresh media on the following day. Viral supernatant was collected 48 and 72 hours later and filtered through a $0.45 \mu \mathrm{m}$ cellulose acetate filter. Viral supernatants were then mixed with polybrene to a final concentration of $8 \mathrm{ng} / \mathrm{mL}$. Adult equine fibroblasts were plated one day prior to transduction at a density of $1 \times 10^{5}$ cells per $100 \mathrm{~mm}$ dish. The cells were incubated overnight with the viral supernatant including equal contributions of the factors and $8 \mathrm{ng} / \mathrm{mL}$ polybrene. The following day, transduction process was performed similar to the first day. A $p M X-G F P$ and no-vector dishes were provided as a positive and negative control, respectively. Transduced cells were then cultured in conventional medium containing $\alpha$-minimum essential medium ( $\alpha$-MEM) with deoxyribonucleosides and ribonucleoside (Invitrogen, Mulgrave, Australia), supplemented with $2 \mathrm{mmol} / \mathrm{mL}$ glutamax (Gibco, Invitrogen, Mulgrave, Australia), 0.1\% (v/v) Mercaptoethanol (Gibco), 1\% $(\mathrm{v} / \mathrm{v})$ nonessential amino acid (NEAA) (Gibco), 1\% (v/v) ITS $(10 \mu \mathrm{g} / \mathrm{mL}$ insulin, $5.5 \mu \mathrm{g} / \mathrm{mL} 125$ transferrin, $6.7 \mathrm{ng} / \mathrm{mL}$ selenium; Gibco), $5 \mathrm{ng} / \mathrm{mL}$ human LIF (Millipore, North Ryde, Australia), 10 ng/mL $\beta$ FGF (Millipore), 10 ng/mL EGF (Invitrogen), $0.5 \%(\mathrm{v} / \mathrm{v})$ penicillin-streptomycin (Gibco), and $20 \%(\mathrm{v} / \mathrm{v})$ FBS. The medium was changed every other day to maintain cell proliferation. After 12 to 16 days of iPS induction, the best colonies based on equine ES celllike colony's morphology were picked and manually passaged onto mouse embryonic fibroblasts (MEFs) inactivated with $4 \mu \mathrm{g} / \mathrm{mL}$ of mitomycin C and plated in an organ culture dish. Colonies were manually cut into small clumps by insulin syringe needles and expanded on the freshly inactivated feeder layers to maintain the EiPS cell line. Seven cell lines were initially produced and maintained in culture, and one cell line was characterised in detail. The transduction efficiency of adult equine fibroblast was evaluated by expression of the $p M X-G F P$ vector control, which was conducted in parallel with the iPS induction experiments. Seventy-two hours after $p M X-G F P$ induction, cells were photographed under a fluorescence microscope, and the percentage of cells expressing GFP was quantified by flow cytometry. Reprogramming efficiency evaluated by correlation of $p M X$ GFP transduction efficiency with iPS cell colony numbers was established [17].

2.1.2. FACS Analysis. Cells were incubated in incubator $\left(37^{\circ} \mathrm{C}, 5 \% \mathrm{Co}_{2}\right)$ using $0.25 \%$ trypsin-EDTA (Invitrogen) for five min and dissociated through pipetting. After spinning at $400 \mathrm{~g}$ for $3 \mathrm{~min}$, the pellet was resuspended and filtered through a $40 \mu \mathrm{m}$ cell strainer (BD Falcon) and analyzed by a BD FACSCanto Flow Cytometer (BD).

\subsection{Characterization of Equine iPS Cell Lines}

2.2.1. Alkaline Phosphatase and Immunofluorescence Staining. Cells were fixed for $15 \mathrm{~min}$ in $4 \%$ (w/v) paraformaldehyde at room temperature and then stained. For alkaline phosphatase (ALP) activity, the cells were stained by histochemistry according to manufacturer's instructions using Alkaline Phosphatase Detection kit (Millipore). For OCT4 and NANOG staining, the cells were permeabilized in $0.2 \%$ Triton X-100 in 3\%(v/v) goat serum in DPBS for $15 \mathrm{~min}$. The cells were incubated with 3\%(v/v) goat serum in DPBS at RT 
TABLE 1: List of primers used for RT-PCR.

\begin{tabular}{lccc}
\hline Markers & \multicolumn{1}{c}{ Primer $\mathrm{F}$} & Primer R & References \\
\hline GAPDH & GATTCCACCCATGGCAAGTTCCATGGCAC & GCATCGAAGGTGGAAGAGTGGGTGTCACT & \\
OCT4 & TCTTTCCACCAGGCCCCCGGCTC & TGCGGGCGGACATGGGGAGATCC & \\
NANOG & TCAAGGACAGGTTTCAGAAGCA & GCTGGGATACTCCACTGGTG & \\
SOX2 & GGTTACCTCTTCCTCCCACTCCAG & TTGCCTTAAACAAGACCACGAAA & Li et al. [12] \\
STAT-3 & TCTGGCTAGACAATATCATCGACCTT & TTATTTCCAAACTGCATCAATGAATCT & Li et al. [12] \\
$\beta$-Tubulin III & CAGAGCAAGAACAGCAGCTACTT & GTGAACTCCATCTCGTCCATGCCCTC & Li et al. [12] \\
GATA-4 & CTCTGGAGGCGAGATGGGACGGG & GAGCGGTCATGTAGAGGCCGGCAGGCATT & Li et al. [12] \\
$\alpha$-Fetoprotein & CTTACACAAAGAAAGCCCCTCAAC & AAACTCCCAAAGCAGCACGAG & Pashaiasl et \\
BMP4 & TCGTTACCTCAAGGGAGTGG & GGCTTTGGGGATACTGGAAT & al \\
\hline
\end{tabular}

OCT4 and SOX2 primers were based on primers specific for Homo sapiens primers. The sequences of these genes were blasted against horse nucleotide sequences that have $95 \%$ and $94 \%$ coverage with the coding sequence of Equus caballus. BMP4 primers were designed on a bovine sequence that has $91 \%$ coverage with the coding sequence of Equallus equa. STAT3, GATA4, $\beta$-tubulin III, and $\alpha$-fetoprotein primers have been applied by Li et al. [12].

for $1 \mathrm{hr}$ to block nonspecific binding of the primary antibodies and then incubated with primary antibodies raised against mouse anti-human SSEA1 (Millipore, MAB4301), mouse anti-human SSEA-4 (Millipore, MAB4304), mouse anti-human OCT4 (Santa Cruz, sc-5279) and rabbit antihuman Nanog (Abcam-ab21603) diluted at 1:100 in DPBS containing $3 \%(\mathrm{v} / \mathrm{v})$ goat serum overnight at $4^{\circ} \mathrm{C}$. The next day the dishes were washed with DPBS three times and incubated with secondary antibodies (diluted in DPBS $1: 1000$, Alexa Flour 594 or 488, Invitrogen) for $1 \mathrm{hr}$ at RT. After three washes with DPBS, the cells were counterstained with $1 \mu \mathrm{g} / \mathrm{mL}$ Hoechst 33342 in DPBS for $10 \mathrm{~min}$ at RT. Control cell lines were treated mouse ESD3 and human ES cells as well as negative control by omitting the primary antibodies (Supplemental Figures 1 and 2) (In Supplementary Material available on line at doi: 10.1155/2012/429160). Images were captured on an Olympus Ix71 microscope.

2.2.2. RNA Extraction and RT-PCR Analysis of Gene Expression. Gene expression was analyzed by RT-PCR. Total RNA was extracted from harvested cell samples using Dynabeads mRNA DIRECT Micro Kit (Invitrogen) or using the RNeasy kit (Qiagen, Doncaster, Australia) according to the manufacturer's instructions. RNA concentrations were determined using the nanoDrop ND-1000 (NanoDrop Technology, Australia). The extracted RNA was treated by RQ1 DNase (Promega, South Sydney, Australia) to remove any contaminating genomic DNA. cDNA was generated using the superscript III enzyme as described before [30]. The first strand CDNA was further amplified by PCR using forward and reverse primers for specific genes. All samples were checked for $G A P D H$ to verify the success of the RT reaction and then for other specific genes with individual primers. PCR amplification was performed in $50 \mu \mathrm{L}$ reaction containing $5 \mu \mathrm{L}$ DNA polymerase $10 \mathrm{x}$ reaction buffer, $3 \mu \mathrm{L}$ $\mathrm{MgCl}_{2}(25 \mathrm{mM}), 1 \mu \mathrm{L}$ dNTP mixture $(10 \mathrm{mM}), 0.4 \mu \mathrm{L}$ GoTaq DNA Polymerase, $1 \mu \mathrm{L}(10 \mu \mathrm{M})$ from each forward and reverse primer, $1 \mu \mathrm{L}$ sample and $\mu \mathrm{L}$ Milli-Q water (Promega). The PCR was processed in a MyCycler Thermal Cycler and run for 35 cycles: denaturation $\left(95^{\circ} \mathrm{C}, 45 \mathrm{~s}\right)$, annealing (55$\left.56^{\circ} \mathrm{C}\right)$, and extension $\left(72^{\circ} \mathrm{C}, 45 \mathrm{~s}\right)$ steps.

All PCR samples were analyzed by electrophoresis on a $2 \%(\mathrm{w} / \mathrm{v})$ agarose gel. The sequence of primers used for PCR and the product size are listed in the Table 1.

2.2.3. Chromosome Counts of Equine iPS Cell Lines. Chromosome counts were performed at P15 and P22. To estimate chromosome number, the cells were treated with 5-bromo2-deoxyuridine (BrdU) overnight and then with Colcemid (Gibco) for a further 4 hours to suppress mitosis. After treating with TrypLE Express (Invitrogen) and hydrating in hypotonic KCL for $15 \mathrm{~min}$, they were washed and fixed in methanol and acetic acid in a ratio of $3: 1$ and centrifuged. The fixation and centrifuge process were repeated three times. The fixed cell pellet was resuspended in $50 \mathrm{uL}$ fixative and was dropped onto clean slides at RT. The slides were stained with a freshly made staining solution containing $3 \mathrm{~mL}$ of Leishman stain in $17 \mathrm{~mL}$ Gurrapostrophes buffer (Invitrogen) for $8 \mathrm{~min}$. The Leishman stain was prepared by dissolving $2 \mathrm{~g}$ Leishman powder in 1 liter methanol. A coverslip was mounted on the slides with Histomount and slides viewed using a light microscope under oil immersion optics (Nikon C1) at 1000x magnification.

\subsection{Differentiation Potential of Equine iPS Cell Line}

2.3.1. Embryoid Body Formation. Equine iPS cells colonies were mechanically dissociated into clumps with needles and cultured on Petri dishes in medium containing $\alpha$-MEM with deoxyribonucleosides and ribonucleoside supplemented with glutamax (Gibco), mercaptoethanol (Gibco), nonessential amino acid (NEAA, Gibco), ITS (insulin, transferrin, selenium; Gibco), penicillin-streptomycin (Gibco), and FBS [30] at $39^{\circ} \mathrm{C}$ in a humidified gas environment of $5 \% \mathrm{CO}_{2}$ in air. Culture medium was changed every 3 days. Samples from attached and nonattached EBs were collected at two weeks to check gene expression of ectodermal markers ( $\beta$-tubulin 

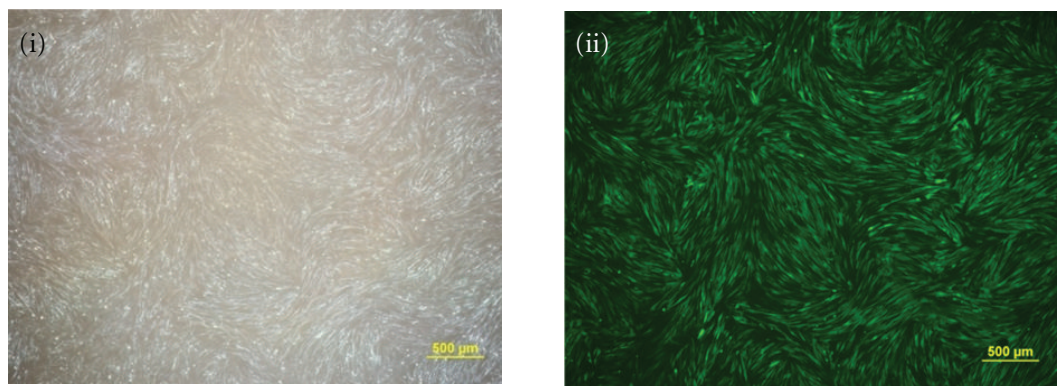

(a)
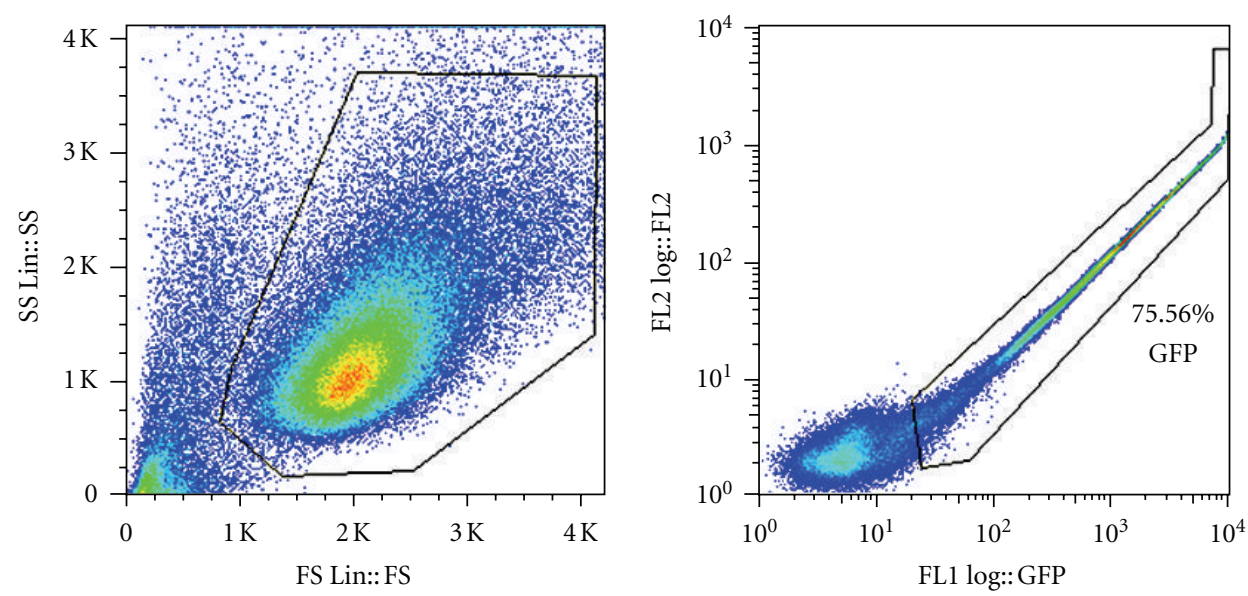

(b)
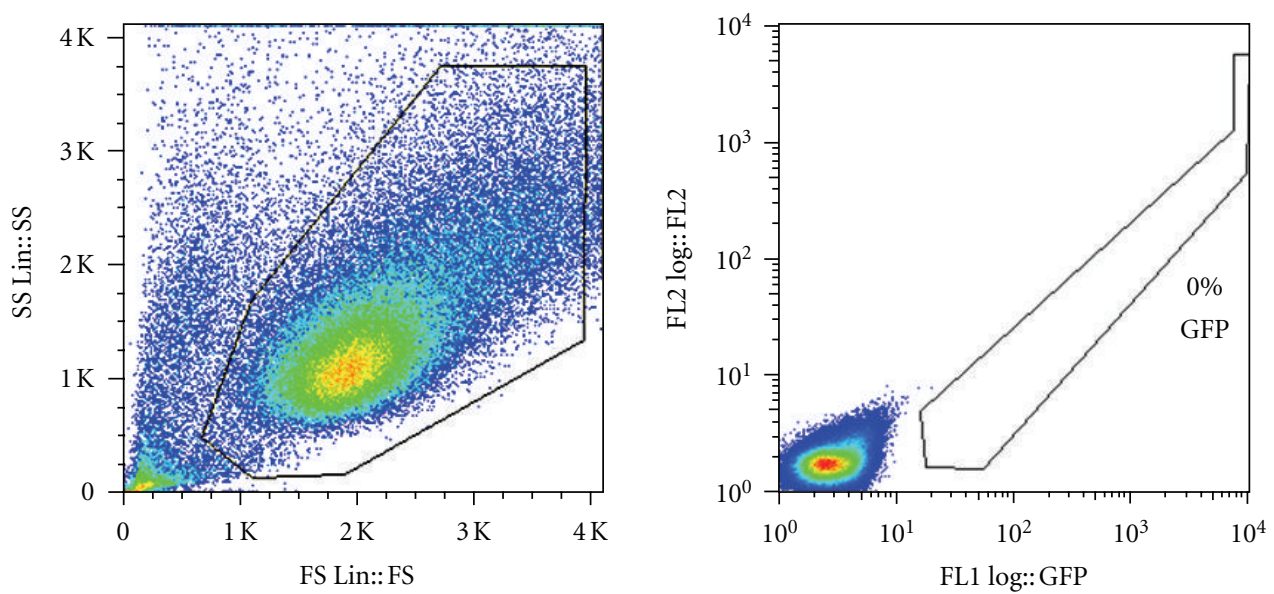

(c)

FIGURE 1: FACs analysis showing GFP fluorescence in adult equine fibroblasts following $p M X$-GFP viral transduction. (a) GFP fluorescence in AEFs following GP2293 mediated retroviral transduction, scale bar $200 \mu \mathrm{M}$. (i) Bright filed. (ii) Green filter. FACs profile of GFP fluorescence, (b) Retroviral transduction using GP2 293 packaging cell. (c) Control EAFs.

III), endodermal markers (Gata4), and mesodermal markers (BMP4) (Table 1) by RT-PCR as described before.

2.3.2. Teratoma Formation. Equine iPS colonies were dissociated into single cells and left on ice until preparation of mice for injection. Five-week-old male SCID mice were used for hind leg muscle injection of $2 \times 10^{6}$ EiPS cells. All procedures were performed with sterile materials in a biological safety cabinet. They were then monitored for well-being and teratoma formation. A growth in the hind leg was visible after approximately 8-10 weeks after injection. Mice were humanely sacrificed; the tumor was dissected out, washed in DPBS, fixed in HistoChoic,e and embedded in paraffin for histological analysis. The samples were sectioned at $4 \mu \mathrm{m}$ thickness onto superfrost slides and allowed to dry overnight. After staining with hematoxylin and eosin, sections were observed using an Olympus Ix71 microscope. 


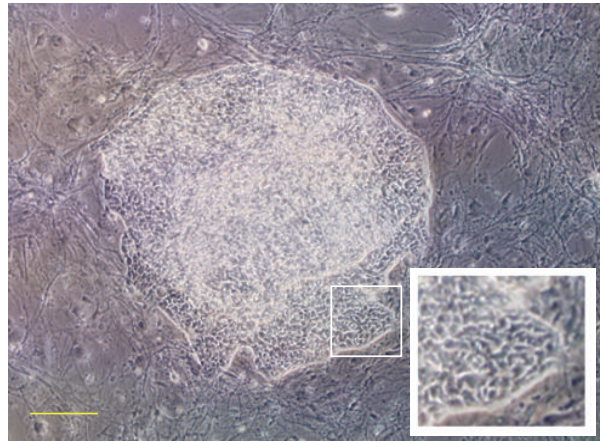

(a)

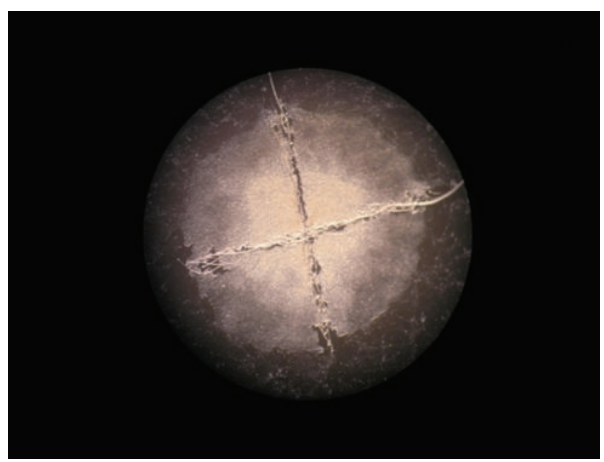

(c)

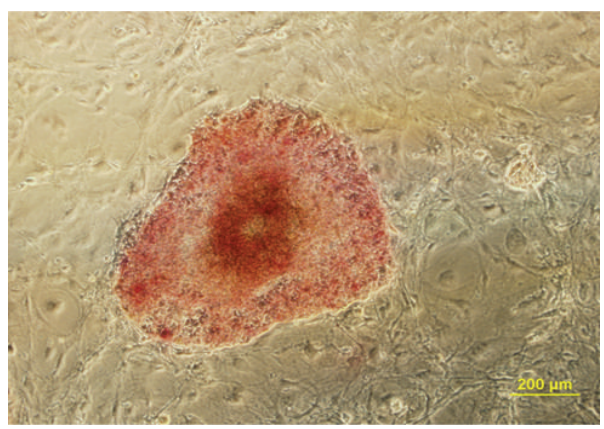

(e)

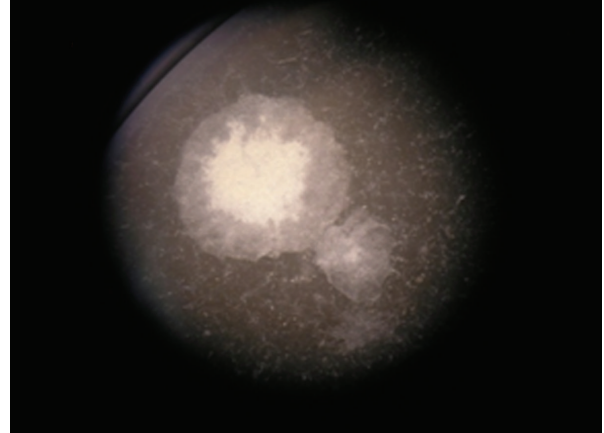

(b)

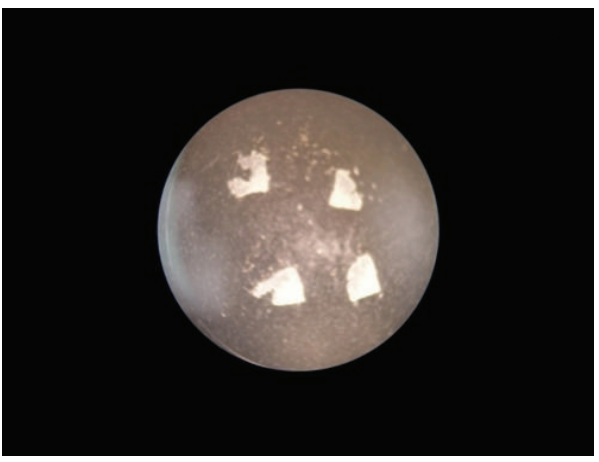

(d)

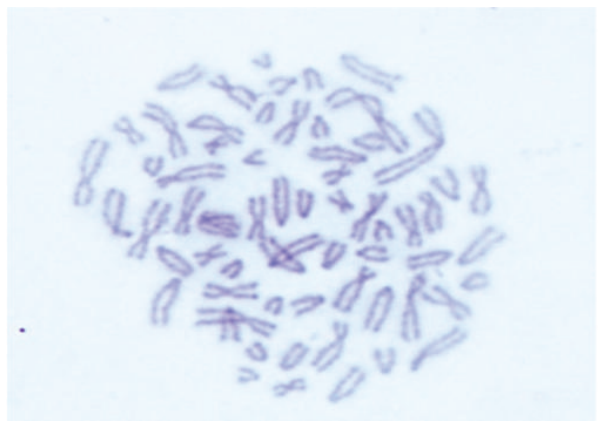

(f)

FIgure 2: Generation of EiPS cells. (a) Morphology of EiPS single colony growing from individual fibroblast. (b) Typical colony of EiPS cells on the MEF and colony selection. (c) Cutting individual colony for manual passaging. (d) Passaged pieces on the MEF. (e) Alkaline phosphatase activity of EiPS cells scale bar $200 \mu \mathrm{M}$. (f) Chromosome spread of EiPS cells.

\section{Freezing and Thawing}

One hour before freezing the cells, a cryofreezing container containing isopropanol was equilibrated at $4^{\circ} \mathrm{C}$. The colonies were dissociated into small clumps about 100 to 200 cells and collected into $15 \mathrm{~mL}$ falcon tube and washed by iPS cells medium and centrifuged for $3 \mathrm{~min}$ at $400 \mathrm{~g}$. Supernatant was discarded, and clumps were resuspended in appropriate amount of EiPS cells medium. Freezing medium which consists of $80 \%$ FBS (JRH Bioscience, Australia) supplemented with $20 \%$ dimethyl sulphoxide (DMSO) was added to prepared $500 \mu \mathrm{L}$ suspension including 80-100 clumps of putative EiPS cells in iPS medium in a cryovial (Nunc, Thermo Fisher, Scoresby, Australia). Then the vials were initially frozen to $-80^{\circ} \mathrm{C}$ overnight and then transferred to a
$\mathrm{LN}_{2}$ tank at minus $196^{\circ} \mathrm{C}$ for long-term storage. The thawing process involved the placing of the cryovials containing the clumps of EiPS cells in a water bath at $37^{\circ} \mathrm{C}$ to be thawed, and cells were transferred to a $15 \mathrm{~mL}$ falcon tube, and then $10 \mathrm{~mL}$ iPS cells medium was slowly added. The cells were centrifuged for $3 \mathrm{~min}$ at $400 \mathrm{~g}$, and then supernatant was discarded, the pallet was resuspended with EiPS medium, and clumps were implanted on fresh MEF in a culture dish using insulin syringe needle.

\section{Results}

4.1. Generation of Induced Pluripotent Stem (iPS) Cells from Adult Equine Fibroblasts. After two rounds of repeated transduction with the three factors (OCT4, SOX2, and Klf4) 


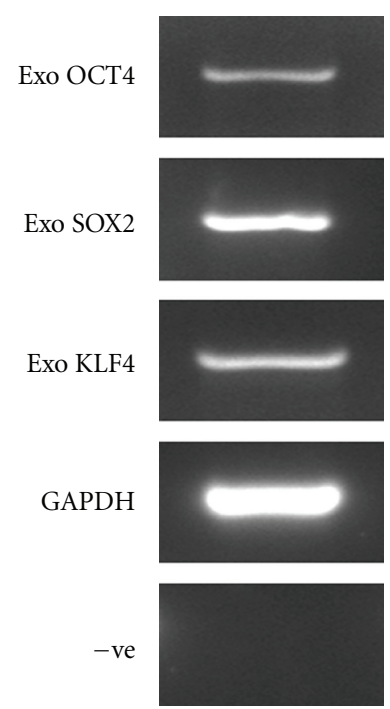

(a)

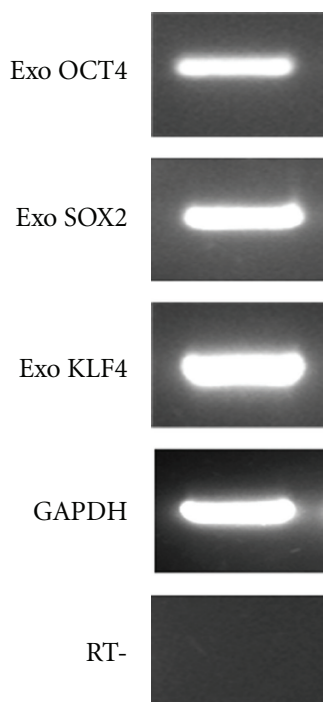

(b)

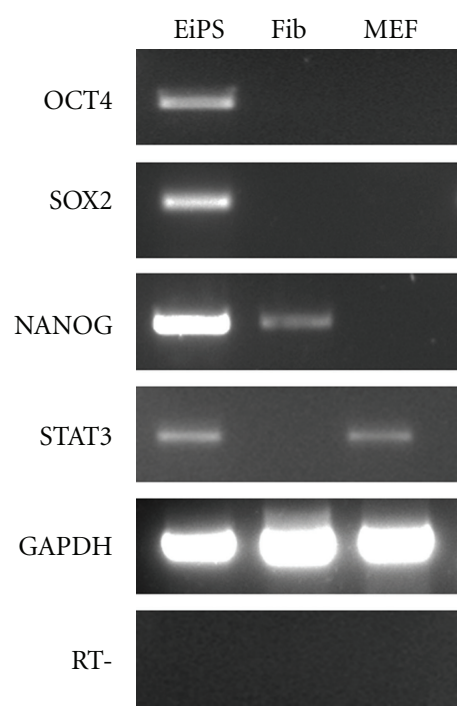

(c)

FIGURE 3: Gene expressions profile of EiPS cells and genomic DNA analysis. (a) Genomic PCR confirming the integration of the four transgenes. (b) Gene expression of exogenous reprogramming factors. (c) Gene expression profile of the EiPS cells compared to the parental EAFs and MEF as feeder cells.

into adult equine fibroblast, we achieved a transduction efficiency of greater than $60 \%$ on day two postinfection using pMX-GFP control plasmid, while negative control showed no GFP-marked cells (Figures 1(b) and 1(c)).

iPS cell colonies first appeared on the day 8-10 postinfection with the dome-like and tightly packed structure. They became large enough at around day 16 to be picked and expanded. Colonies were isolated mechanically and transferred onto prepared culture dishes containing MEF layer and equine ES cell medium (Figure 2).

\subsection{Characterization of Equine iPS Cell Line. EiPS cells had a} low cytoplasm to nuclear ratio and formed colonies to those observed in cattle [28]. The cell line was characterized by molecular analysis. The integration of reprogramming transgenes into the genome of the cells was confirmed by gDNA PCR analysis and expression of exogenous factor examined at passage 24 (Figures 3(a) and 3(b)). RT-PCR analysis showed mRNA expression of key pluripotent markers including OCT4, SOX2, NANOG, and STAT3 (Figure 3(c)). Some expression of Nanog was detected in equine fibroblasts, and STAT3 was also detected in the mouse embryonic feeder cells using the primer pairs. The cell line expressed a high level of alkaline phosphatase activity (Figure 2(e)). They were positive for protein expression of OCT4, NANOG, SSEA1, and SSEA4 as determined by immunofluorescent staining (Figure 4). Moreover, chromosome spreads revealed a normal diploid chromosome count of 64 in metaphase spreads at passage 15 (data not shown) and 22 (Figure 2(f)). More than $90 \%$ of frozen EiPS cells clumps were recovered after thawing and formed colonies after implanting on fresh MEF feeder layer. Thawed cell lines survived and were maintained for more than four passages without losing iPS cell morphology.
4.2.1. Differentiation Potential of Equine iPS Cells. The EiPS cells formed embryoid bodies after 5 days in suspension culture, after which they were transferred to gelatin-coated dishes to attach and develop outgrowths (Figures 5(a) and 5(b)). RT-PCR results demonstrated mRNA expression of genes representative of the three embryonic germ layers $[11,12]$, endoderm ( $\alpha$-fetoprotein), mesoderm (Gata4 and BMP4), and ectoderm ( $\beta$-tubulinIII) (Figure 5(c)). Equine iPS cells formed teratomas 8 to 10 weeks after injection containing cells of the three embryonic germ layers: endoderm (vessels), mesodermal cells (muscle), and ectoderm (epidermal cells) (Figure 5(d)).

\section{Discussion}

Due to similarity in size, physiology, and immunology, large animals are better models for human genetic or acquired diseases compared with rodents. In addition, they have a longer life span and have a heterogeneous genetic background which is similar to humans and unlike rodents; therefore, they can provide a good model for long-term experiments. Also about 95 equine genetic diseases share a high homology with human genetic defects [13]. Furthermore, equine can be an appropriate model for human diseases such as osteoarthritis as well as a model for musculoskeletal injuries as there are common features of the athletic injuries in human and equine. Limited capability for full functional repair of musculoskeletal injuries has limited treatments outcomes [1]. Joint injuries and related illnesses cost an estimated US $\$ 6.5$ billion annually for the equine race industry [13].

MSCs, ESCs, and iPS cells are options for research and therapeutic applications regarding musculoskeletal injuries. Compared with MSCs and ESCs, iPS cells are better as they 

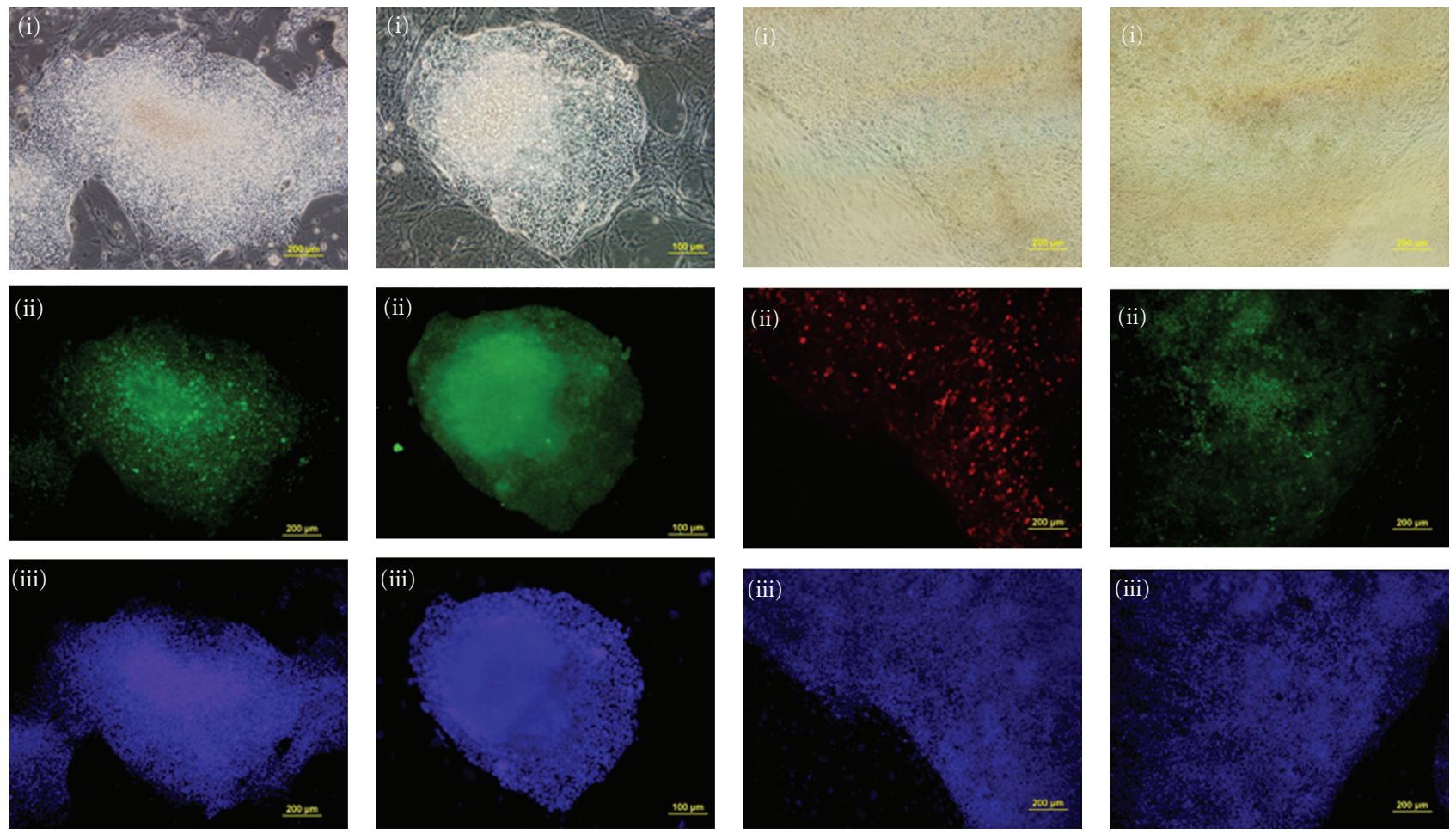

(a)

(b)

(c)

(d)

FIGURE 4: Immunoflourescence staining of pluripotent markers in EiPS cells. Immunostaining of EiPS cells for (a) OCT4, (b) NANOG, (c) SSEA1, and (d) SSEA4, counterstained with DAPI, scale bar $200 \mu \mathrm{M}$.

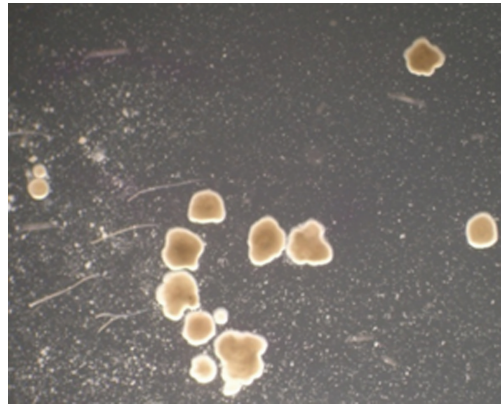

(a)

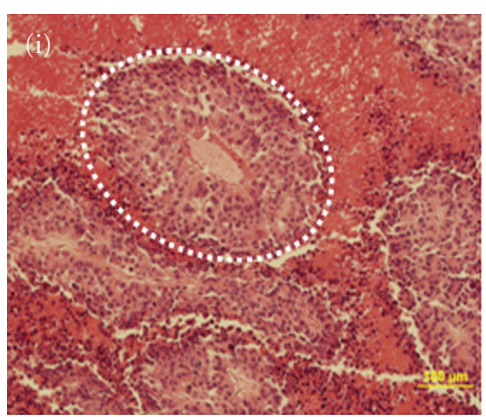

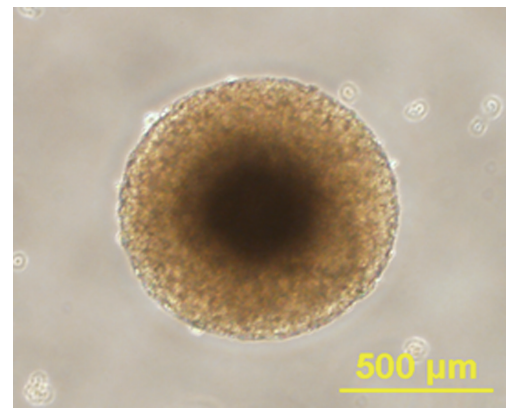

(b)

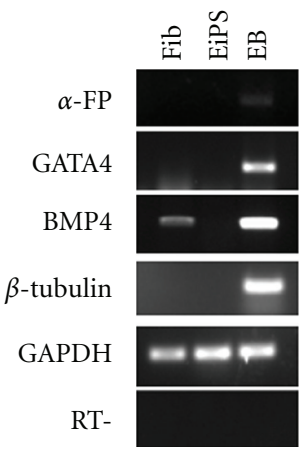

(c)

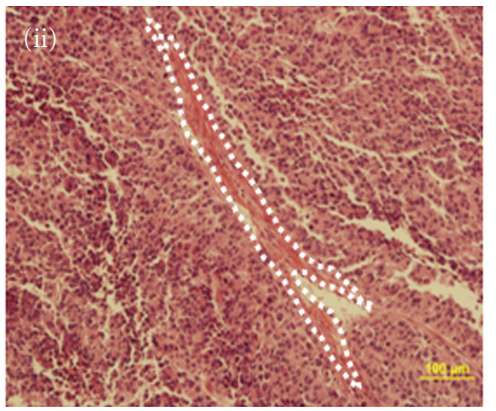

(d)

FIGURE 5: Differentiation potential of EiPS cells. (a) Embryoid body formation of EiPS cells grown in suspension medium in the absence of LIF. (b) Single EB, scale bar $500 \mu \mathrm{M}$. (c) Gene expression profile of EiPS cells following differentiation of embryoid body. (d) Histology of differentiated tissues found in the hind leg muscle of SCID mice following injection of EiPS cells included (i) endodermal differentiation, (ii) mesodermal differentiation, (iii) ectodermal (neuroblastic) differentiation, scale bar $100 \mu \mathrm{M}$. 
provide pluripotent cells that can be immunocompatible to the recipient. There is one report on induction of pluripotency in equine [29], using the Yamanaka cocktail (OSKM) to generate iPS cells from fetal cells. In this study we report the generation of equine iPS cells from adult cells and without the use of the protooncogene c-MYC which opens the door for autologous transplantation in cartilage and tendon injury models. Similar to the finding of Nagy and colleagues the equine iPS cells generated required continuous expression of the transgenes to maintain pluripotency. Apart from one report in sheep [21], iPS cells generated in other domestic species have shown similar traits $[12,18$, $24,25,28$ ], suggesting that maintenance of pluripotency largely depends on the expression of the reprogramming transgenes.

We established the equine iPS cell line which proliferated in culture beyond 27 passages. The cells maintained ESC characteristics and expressed pluripotent markers including alkaline phosphatase activity and expression of pluripotency markers OCT4 and NANOG. Furthermore, the cells stained positively for SSEA1 similar to mouse pluripotent cells; as well as SSEA4 which is expressed on human pluripotent cells, similar findings have been reported in equine ES $[11,12]$ and iPS cells [29]. The EiPS cells expressed pluripotency genes OCT4, SOX2, NANOG, and STAT3 by RT-PCR. The EiPS cells showed differentiation potential in vitro by EB formation and expressing genes indicative of the three embryonic germ layers. Some of the discrepancies in the markers are due to the difficulties in characterizing pluripotency in the horse as there is a lack of reliable pluripotency markers [1] and lack of suitable antibodies raised against equine cells for immunocytochemical analyses [29]. Therefore, in vivo differentiation by teratoma formation was used as further evidence of pluripotential of the cells as has been routinely conducted for iPS cells from most domestic species.

In summary, our findings indicate that adult equine fibroblast can be reprogrammed into pluripotent state via the retroviral delivery of transcription factors, OCT4, SOX2, and KLF4. The generated iPS cells are pluripotent as shown by expression of pluripotent markers and have capability to differentiate into cell types indicative of the three embryonic germ layers both in vitro and in vivo.

\section{Acknowledgment}

This project was supported by the Victorian Government's Operational Infrastructure Support Program. K. Khodadadi and H. Sumer contributed equally to the work.

\section{References}

[1] D. B. B. P. Paris and T. A. E. Stout, "Equine embryos and embryonic stem cells: defining reliable markers of pluripotency," Theriogenology, vol. 74, no. 4, pp. 516-524, 2010.

[2] K. C. Kemp, J. Hows, and C. Donaldson, "Bone marrowderived mesenchymal stem cells," Leukemia and Lymphoma, vol. 46, no. 11, pp. 1531-1544, 2005.

[3] M. L. Weiss and D. L. Troyer, "Stem cells in the umbilical cord," Stem Cell Reviews, vol. 2, no. 2, pp. 155-162, 2006.
[4] M. N. Helder, M. Knippenberg, J. Klein-Nulend, and P. I. J. M. Wuisman, "Stem cells from adipose tissue allow challenging new concepts for regenerative medicine," Tissue Engineering, vol. 13, no. 8, pp. 1799-1808, 2007.

[5] Y. S. Yoon, N. Lee, and H. Scadova, "Myocardial regeneration with bone-marrow-derived stem cells," Biology of the Cell, vol. 97, no. 4, pp. 253-263, 2005.

[6] H. L. Holtorf, T. L. Sheffield, C. G. Ambrose, J. A. Jansen, and A. G. Mikos, "Flow perfusion culture of marrow stromal cells seeded on porous biphasic calcium phosphate ceramics," Annals of Biomedical Engineering, vol. 33, no. 9, pp. 1238-1248, 2005.

[7] M. E. Bernardo, J. A. M. Emons, M. Karperien et al., "Human mesenchymal stem cells derived from bone marrow display a better chondrogenic differentiation compared with other sources," Connective Tissue Research, vol. 48, no. 3, pp. 132140, 2007.

[8] R. K. W. Smith, M. Korda, G. W. Blunn, and A. E. Goodship, "Isolation and implantation of autologous equine mesenchymal stem cells from bone marrow into the superficial digital flexor tendon as a potential novel treatment," Equine Veterinary Journal, vol. 35, no. 1, pp. 99-102, 2003.

[9] S. Wakitani, K. Imoto, T. Yamamoto, M. Saito, N. Murata, and M. Yoneda, "Human autologous culture expanded bone marrow-mesenchymal cell transplantation for repair of cartilage defects in osteoarthritic knees," Osteoarthritis and Cartilage, vol. 10, no. 3, pp. 199-206, 2002.

[10] S. Violini, P. Ramelli, L. F. Pisani, C. Gorni, and P. Mariani, "Horse bone marrow mesenchymal stem cells express embryo stem cell markers and show the ability for tenogenic differentiation by in vitro exposure to BMP-12," BMC Cell Biology, vol. 10, article no. 29, 2009.

[11] S. Saito, H. Ugai, K. Sawai et al., "Isolation of embryonic stemlike cells from equine blastocysts and their differentiation in vitro," FEBS Letters, vol. 531, no. 3, pp. 389-396, 2002.

[12] X. Li, S. G. Zhou, M. P. Imreh, L. Ährlund-Richter, and W. R. Allen, "Horse embryonic stem cell lines from the proliferation of inner cell mass cells," Stem Cells and Development, vol. 15, no. 4, pp. 523-531, 2006.

[13] R. T. Tecirlioglu and A. O. Trounson, "Embryonic stem cells in companion animals (horses, dogs and cats): present status and future prospects," Reproduction, Fertility and Development, vol. 19, no. 6, pp. 740-747, 2007.

[14] H. Lin, J. Lei, D. Wininger et al., "Multilineage potential of homozygous stem cells derived from metaphase II oocytes," Stem Cells, vol. 21, no. 2, pp. 152-161, 2003.

[15] I. Wilmut, N. Beaujean, P. A. De Sousa et al., "Somatic cell nuclear transfer," Nature, vol. 419, no. 6907, pp. 583-586, 2002.

[16] T. Wakayama, V. Tabar, I. Rodriguez, A. C. F. Perry, L. Studer, and P. Mombaerts, "Differentiation of embryonic stem cell lines generated from adult somatic cells by nuclear transfer," Science, vol. 292, no. 5517, pp. 740-743, 2001.

[17] K. Takahashi and S. Yamanaka, "Induction of pluripotent stem cells from mouse embryonic and adult fibroblast cultures by defined factors," Cell, vol. 126, no. 4, pp. 663-676, 2006.

[18] K. Okita, T. Ichisaka, and S. Yamanaka, "Generation of germline-competent induced pluripotent stem cells," Nature, vol. 448, no. 7151, pp. 313-317, 2007.

[19] M. Wernig, A. Meissner, R. Foreman et al., "In vitro reprogramming of fibroblasts into a pluripotent ES-cell-like state," Nature, vol. 448, no. 7151, pp. 318-324, 2007. 
[20] J. Yu, M. A. Vodyanik, K. Smuga-Otto et al., "Induced pluripotent stem cell lines derived from human somatic cells," Science, vol. 318, no. 5858, pp. 1917-1920, 2007.

[21] J. Liu, D. Balehosur, B. Murray, J. M. Kelly, H. Sumer, and P. J. Verma, "Generation and characterization of reprogrammed sheep induced pluripotent stem cells," Theriogenology, vol. 77, no. 2, pp. 338-346.e1, 2012.

[22] W. Li, W. Wei, S. Zhu et al., "Generation of rat and human induced pluripotent stem cells by combining genetic reprogramming and chemical inhibitors," Cell Stem Cell, vol. 4, no. 1, pp. 16-19, 2009.

[23] J. Liao, C. Cui, S. Chen et al., "Generation of induced pluripotent stem cell lines from adult rat cells," Cell Stem Cell, vol. 4, no. 1, pp. 11-15, 2009.

[24] M. A. Esteban, J. Xu, J. Yang et al., "Generation of induced pluripotent stem cell lines from Tibetan miniature pig," Journal of Biological Chemistry, vol. 284, no. 26, pp. 17634 17640, 2009.

[25] T. Ezashi, B. P. V. L. Telugu, A. P. Alexenko, S. Sachdev, S. Sinha, and R. M. Roberts, "Derivation of induced pluripotent stem cells from pig somatic cells," Proceedings of the National Academy of Sciences of the United States of America, vol. 106, no. 27, pp. 10993-10998, 2009.

[26] F. D. West, S. L. Terlouw, D. J. Kwon et al., "Porcine induced pluripotent stem cells produce chimeric offspring," Stem Cells and Development, vol. 19, no. 8, pp. 1211-1220, 2010.

[27] Z. Wu, J. Chen, J. Ren et al., "Generation of pig induced pluripotent stem cells with a drug-inducible system," Journal of molecular cell biology, vol. 1, no. 1, pp. 46-54, 2009.

[28] H. Sumer, J. Liu, L. F. Malaver-Ortega, M. L. Lim, K. Khodadadi, and P. J. Verma, "NANOG is a key factor for induction of pluripotency in bovine adult fibroblasts," Journal of Animal Science, vol. 89, no. 9, pp. 2708-2716, 2011.

[29] K. Nagy, H. -K. Sung, P. Zhang et al., "Induced pluripotent stem cell lines derived from equine fibroblasts," Stem Cell Reviews and Reports, vol. 7, no. 3, pp. 693-702, 2011.

[30] M. Pashaiasl, K. Khodadadi, M. K. Holland, and P. J. Verma, "The efficient generation of cell lines from bovine parthenotes," Cellular Reprogramming, vol. 12, no. 5, pp. 571-579, 2010. 

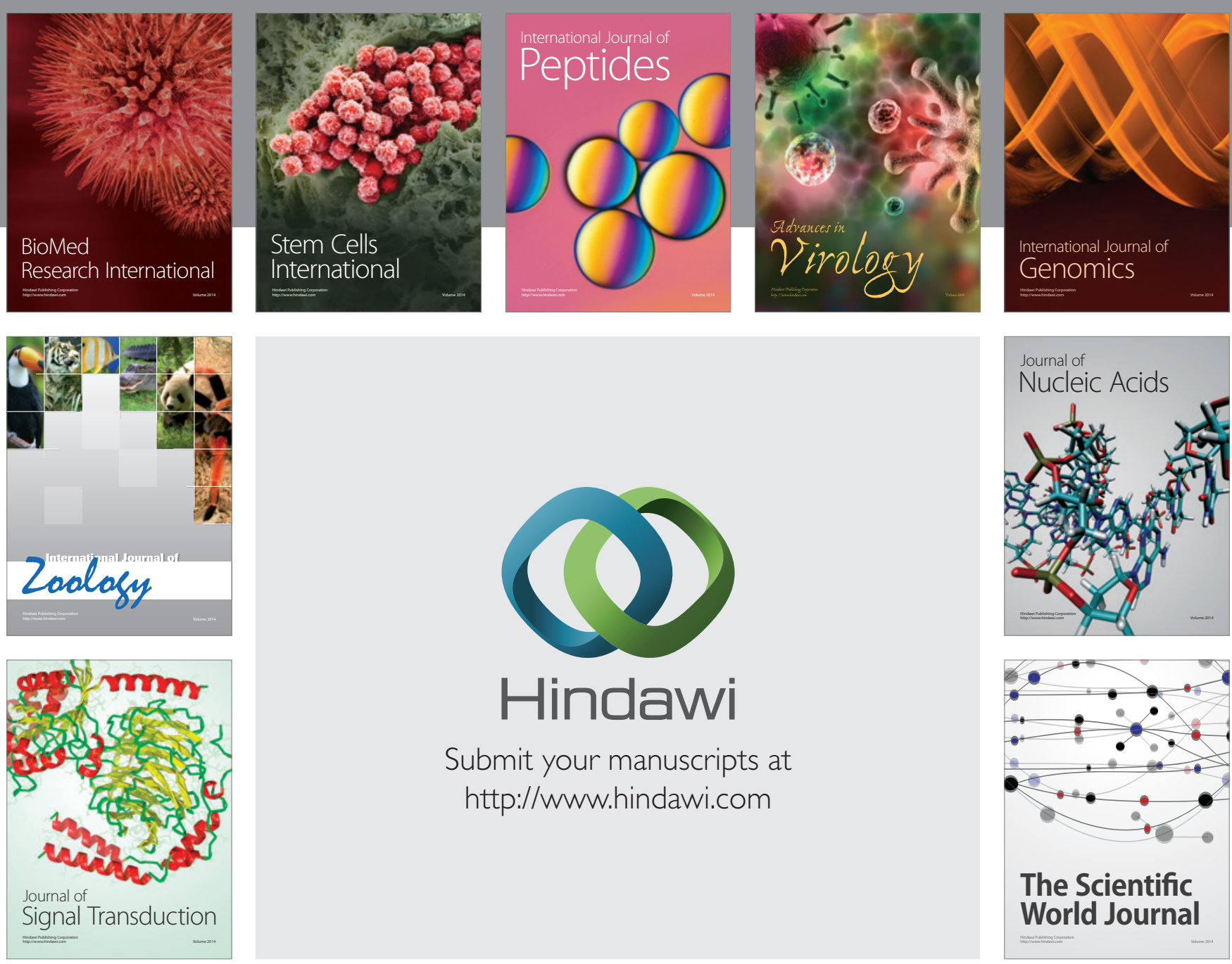

Submit your manuscripts at

http://www.hindawi.com
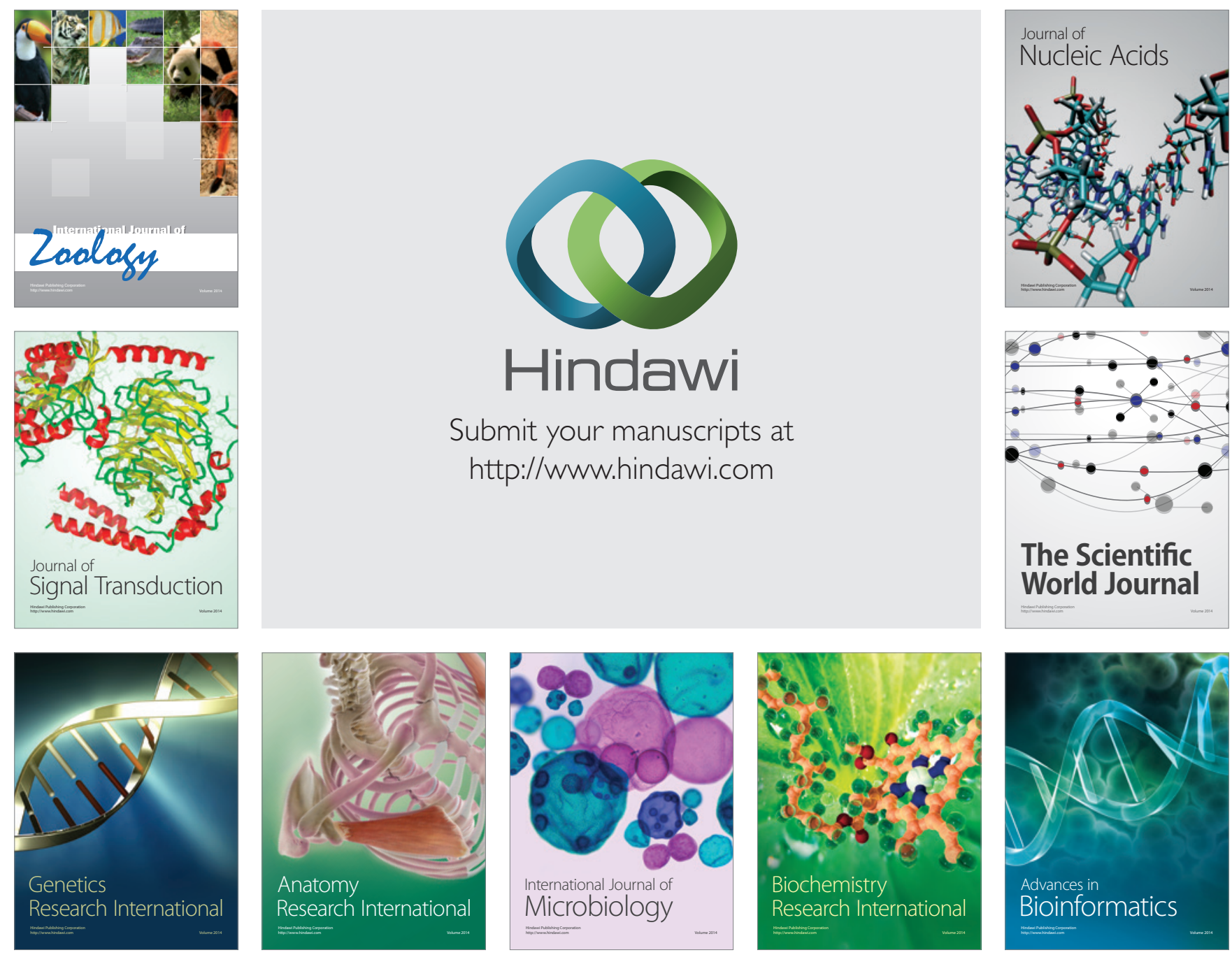

The Scientific World Journal
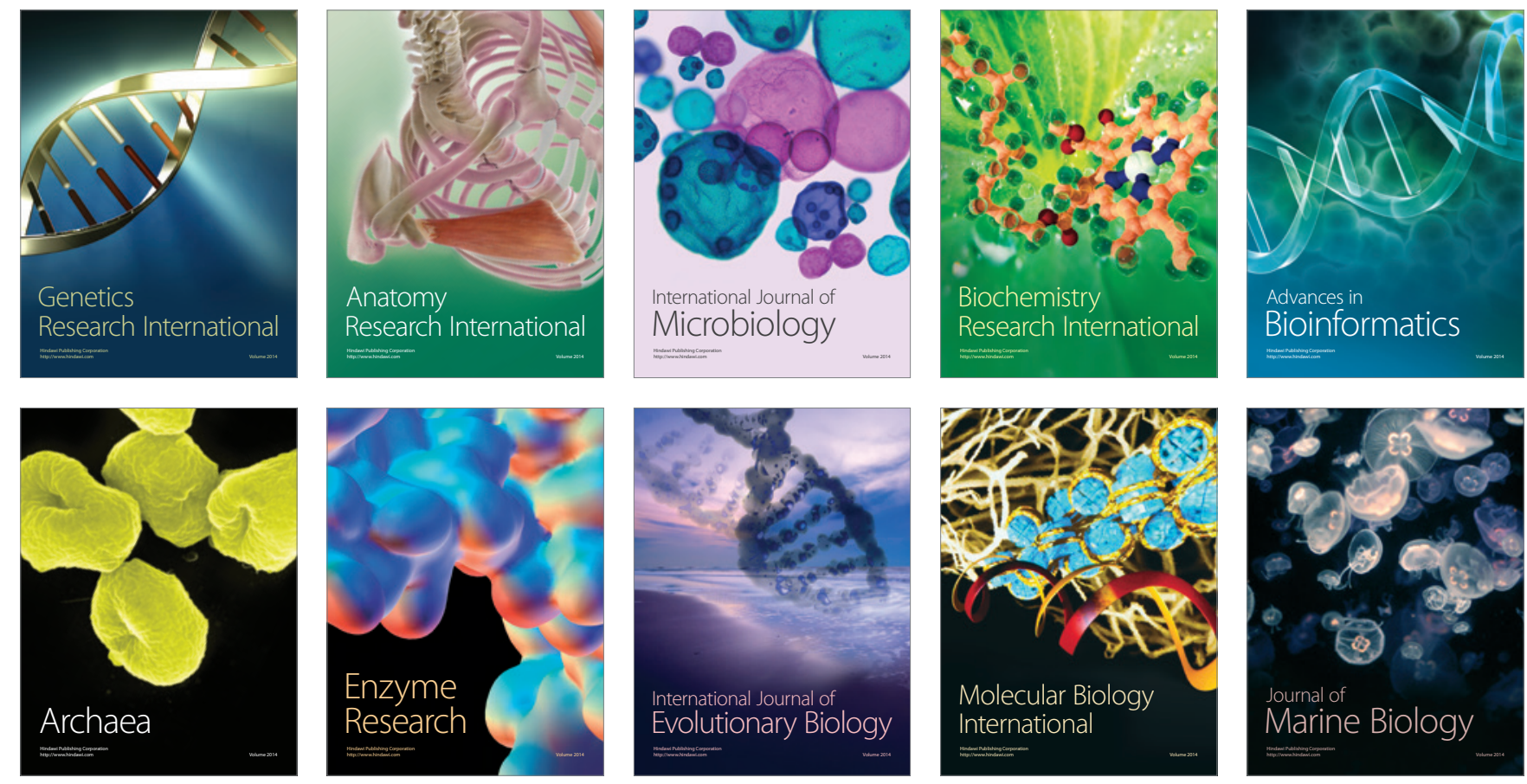